\title{
Physical parameters of contact binaries through 2-D and 3-D correlation diagrams
}

\author{
K. D. Gazeas \\ Harvard-Smithsonian CfA, 60 Garden Street, Cambridge, MA 02138, USA
}

\begin{abstract}
Physical parameters of contact binaries are studied through 2-D and 3-D correlations among them. It is shown that the physical parameters (i.e. mass, radius and luminosity) are closely correlated with the orbital period and mass ratio in the 3-D domain. These correlations can be used as a quality check for the parameters in every given solution of a contact binary. The empirical laws, extracted out of these correlations, are a useful tool for a quick estimate of physical parameters for the numerous contact binaries found in global sky surveys.
\end{abstract}

\section{The 2-D and 3-D correlation diagrams}

Our sample is based on the list of 112 contact binaries published by Gazeas \& Stepien (2008). These binaries are the only systems with accurate solutions, based on high quality photometric light curves and good radial velocity curves for both components. We show that several relations and correlations exist among the discussed parameters. Some of them may be useful in the future for approximate estimates of masses and radii of contact binaries for which the orbital period is known. In our study we consider component " 1 " as the presently more massive one. Our assumption is based on the double-lined spectroscopic observations, where the mass ratio is taken as $q=M_{2} / M_{1} \leq 1$.

As shown also by Gazeas \& Niarchos (2006), the plots of mass and angular momentum versus orbital period gave a direct evidence of evolution into contact, as well as the evolution of A-type W UMa binaries towards the W-type. Figure 1 (upper panel) shows a plot of mass versus orbital period, as well as the equivalent plots of radius and luminosity in logarithmic scale. It was easy to extract empirical laws out of these diagrams, as the correlation of absolute parameters with the orbital period is more than obvious, with an error of approximately $15 \%$ (Eqs. 1-6). In the lower panel, the same physical parameters are plotted against orbital period and mass ratio as: $M=M(P, q), R=R(P, q), L=L(P, q)$. The extracted empirical laws (Eqs. 7-12) give an error less than $5 \%$.

\section{Conclusions}

The errors derived from the 3-D correlation equations are significantly small, especially in the prediction of mass and radius. The determination of the physical parameters from the above diagrams is independent of the orbital inclination and (possible) presence of additional objects in a system, derived from the light curve. It is so accurate that it can be used in the opposite way, checking the solutions obtained with the classic procedure. The importance of such an approximation is that we will be able to calculate directly the absolute parameters 

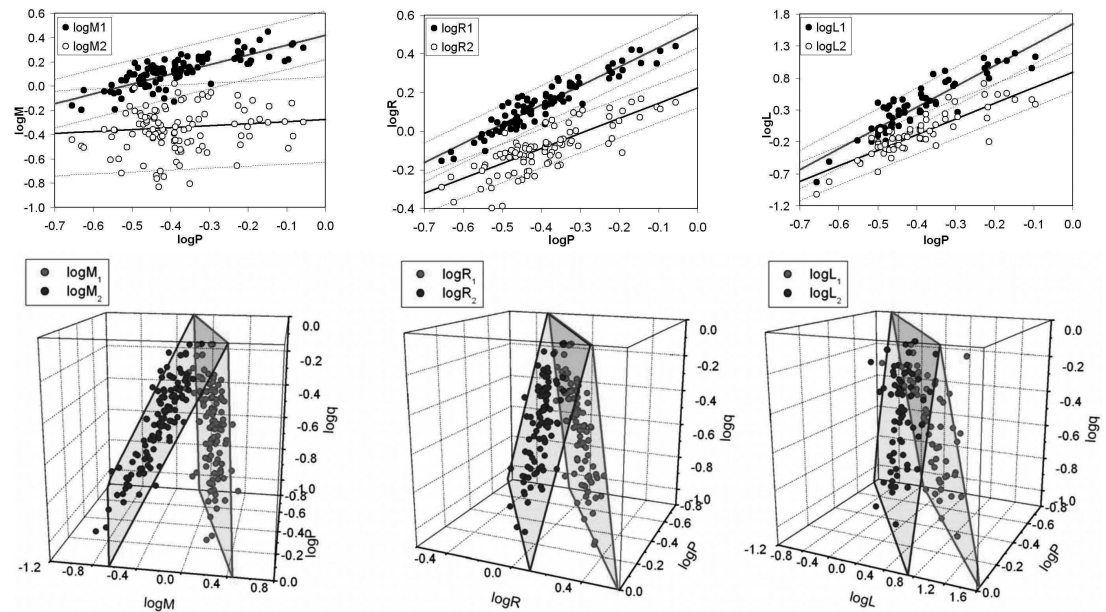

Figure 1: The 2-D (upper panel) and 3-D (lower panel) correlation diagrams for the absolute physical parameters $M, R$ and $L$, as described in the text by Eqs. 1-6 and 7-12, respectively.

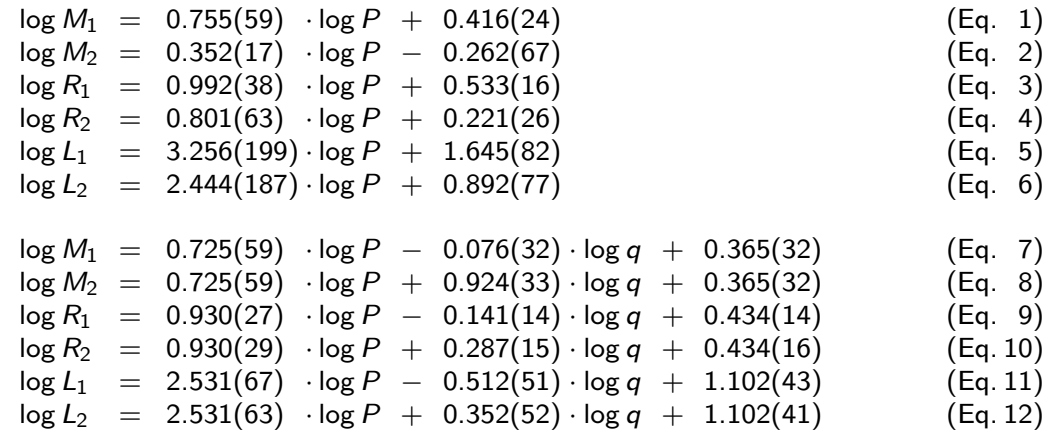

of such systems, knowing only their fundamental observational characteristics (orbital period and mass ratio). This will provide a great opportunity to study easily and much faster the properties of thousands of contact binaries that were discovered (or will be discovered soon) by the global sky surveys. On the other hand, the absolute parameters will be used as distance indicators, since contact binaries have without doubt many advantages as standard candles (Rucinski 1996). It is important to note that the 2-D and 3-D empirical laws do not give the full solution of contact binary systems. They do give a very accurate approximation of their physical parameters, as well as a very good view of the contact binary systems' structure.

\section{References}

Gazeas, K., \& Niarchos, P. 2006, MNRAS, 370, 29

Gazeas, K., \& Stepien, K. 2008, MNRAS, 390, 1577

Rucinski, S. M. 1996, ASP Conf. Ser., 90, 270 